EPJ Web of Conferences 43, 04009 (2013)

DOI: $10.1051 /$ epjconf/20134304009

(C) Owned by the authors, published by EDP Sciences, 2013

\title{
Erratum to: A survey of hot subdwarf pulsators in $\omega$ Cen
}

\author{
S.K. Randall1,a , A. Calamida², G. Fontaine ${ }^{3}$, E.M. Green ${ }^{4}$, M. Monelli ${ }^{5,6}$, \\ M.L. Alonso ${ }^{7}$, M. Catelan 8,9 , G. Bono ${ }^{10}$, V.S. Dhillon ${ }^{11}$ and T.R. Marsh ${ }^{12}$ \\ ${ }^{1}$ ESO, Karl-Schwarzschild-Str. 2, 85748 Garching bei München, Germany \\ ${ }^{2}$ Osservatorio Astronomico di Roma, Istituto Nazionale de Astrofisica, via Frascati 33, \\ 0040 Monte Porzio Catone, Italy \\ ${ }^{3}$ Département de Physique, Université de Montréal, CP. 6128, Succ. Centre-Ville, Montréal, \\ QC H3C 3J7, Canada \\ ${ }^{4}$ Steward Observatory, University of Arizona, 933 North Cherry Avenue, Tucson, \\ AZ 85721, USA \\ ${ }^{5}$ Instituto de Astrofísica de Canarias, Calle Via Lactea s/n, 38205 La Laguna, Tenerife, Spain \\ ${ }^{6}$ Departamento de Astrofísica, Universidad de La Laguna, Tenerife, Spain \\ ${ }^{7}$ Instituut voor Sterrenkunde, KU Leuven, Celestijnenlaan 200D, 3001 Leuven, Belgium \\ ${ }^{8}$ Departamento de Astronomía y Astrofísica, Pontificia Universidad Católica de Chile, \\ Av. Vicuña Mackenna 4860, 782-0436 Macul, Santiago, Chile \\ ${ }^{9}$ The Milky Way Millennium Nucleus, Av. Vicuña Mackenna 4860, 782-0436 Macul, \\ Santiago, Chile \\ ${ }^{10}$ Department of Physics, Università di Roma "Tor Vergata", via della Ricerca Scientifica 1, \\ 00133 Rome, Italy \\ ${ }^{11}$ Department of Physics and Astronomy, University of Sheffield, Sheffield S3 7RH, UK \\ ${ }^{12}$ Department of Physics, University of Warwick, Coventry CV4 7AL, UK
}

Original article:

EPJ Web of Conferences 43, 04006 (2013), DOI: 10.1051/epjconf/20134304006

The name of the first author should be S.K. Randall.

\footnotetext{
${ }^{a}$ e-mail: srandall@eso.org
}

This is an Open Access article distributed under the terms of the Creative Commons Attribution License 2.0, which permits unrestricted use, distribution, and reproduction in any medium, provided the original work is properly cited. 